retrievers compared to thromboaspiration $(40.7 \%$ against $27.6 \%)$, there was no statistically relevant difference $(p=0,165)$. Thromboaspiration as the first-line reperfusion method was associated with more complete (mTICI 3, $74.1 \%$ ) and successful (mTICI 2b-3, 86.5\%) reperfusion by the end of the intervention. The use of stent retrievers as the first-line method led to less frequent complete $(55.6 \%)$ $(p=0,048)$ and successful $(66.6 \%) \quad(p=0,009)$ reperfusion. The efficiency of the reperfusion in posterior circulation depends on the first-line method and the number of passes. Thus, after the first pass with a stent retriever the level of successful $(p=0.04)$ and complete $(p=0.015)$ reperfusion is statistically lower than with thromboaspiration. SAVE technique also proved to be effective in achieving more successful $(p=0.015)$ and complete $(p=0.003)$ reperfusion comparing to stent retrievers. However, thrombectomy with a stent retriever as a second-line method of recanalization was used more often than thromboaspiration or SAVE. The second-line method was ineffective in $25.8 \%$ of cases, which required switching to the third method of reperfusion. This figure is comparable to the need to switch to the second-line reperfusion method in 29.4\% of cases.

Disclosure Nothing to disclose

\section{EP61 DIRECT CAROTID PUNCTURE FOR MECHANICAL THROMBECTOMY IN ACUTE ISCHAEMIC STROKE: A SINGLE CENTRE EXPERIENCE AND REVIEW OF THE LITERATURE}

JCY Chan, IHW Cheung, YLE Chu, R Lee. Department of Radiology, Queen Mary Hospital, Hong Kong, Hong Kong

\subsection{6/neurintsurg-2021-ESMINT.60}

Introduction Direct carotid puncture (DCP) as a bailout or primary vascular access technique for endovascular thrombectomy (EVT) has been sporadically described in the literature. The collective procedural risk profile and therapeutic outcomes remain unclear.

Objectives and Aims To establish the efficacy and safety profile of DCP.

Method We reviewed our prospectively maintained singlecentre database of patients admitted for acute ischaemic stroke (AIS) who underwent EVT. 11 patients treated by DCP approach were identified. We also conducted a literature review on published cases of EVT performed via DCP.

Results 9 studies with a total of 106 cases (our data included) were reviewed. Initial NIHSS score ranges from 2 to 31 (average 17.1). DCP access was successful in 92.5\%. Among this, $86 \%$ achieved satisfactory recanalization (mTICI $\geq 2 \mathrm{~b}$ ). Average post-procedural $\mathrm{mRS}$ is 3.8. Carotid access sites were managed with closure devices in $76.6 \%$, with Angioseal being the most commonly deployed device. Haemostasis was achieved by manual compression or combined method in $22.2 \%$ and $5.1 \%$ of the cases respectively.

Carotid access site-related complications were encountered in 19 cases (17.9\%). These include puncture site haematoma $(n=12)$, non-flow-dependent carotid artery dissection $(n=4)$, access site pseudoaneurysm $(n=2)$ and retinal artery occlusion $(n=1) .4$ cases required further intervention(3.8\%). No mortality related to access site complication was reported.

Conclusion Direct carotid puncture is an effective and generally safe approach for EVT, with major access-site related complications seen in $<5 \%$ of the cases. It should be considered as a bailout technique or primary access approach in selected cases.

\section{REFERENCES}

1. Akpinar CK, et al. Direct common carotid artery puncture: rescue mechanica thrombectomy strategy in acute ischemic stroke. Neurointervention 2020;15 (2):60-66.

2. Cord BJ, et al. Direct carotid puncture for mechanical thrombectomy in acute ischemic stroke patients with prohibitive vascular access. I Neurosurg 2020:1-11.

3. Miszczuk M, et al. Direct puncture of the carotid artery as a bailout vascular access technique for mechanical thrombectomy in acute ischemic stroke-the revival of an old technique in a modern setting. Neuroradiology 2021;63(2):275-283.

Disclosure Nothing to disclose

\section{EP62 ENDOVASCULAR TRANSCAROTID ARTERY REVASCULARIZATION USING THE WALRUS BALLOON GUIDE CATHETER: SAFETY AND FEASIBILITY FROM MULTICENTER EXPERIENCE}

${ }^{1} \mathrm{M}$ Salem, ${ }^{2} \mathrm{C}$ Griessenauer, ${ }^{1} \mathrm{~S}$ Kvint, ${ }^{3} \mathrm{~A}$ Baig, ${ }^{3} \mathrm{~A}$ Monteiro, ${ }^{4} \mathrm{G}$ Cortez, ${ }^{5} \mathrm{~A}$ Kuhn, ${ }^{1} \mathrm{O}$ Choudhri, ${ }^{2} \mathrm{O}$ Goren, ${ }^{2} \mathrm{~S}$ Dalal, ${ }^{6} \mathrm{P}$ Jabbour, ${ }^{7} \mathrm{RM}$ Starke, ${ }^{5} \mathrm{~A}$ Puri, ${ }^{4} \mathrm{R}$ Hanel, ${ }^{3} \mathrm{El}$ Levy, ${ }^{3}$ A Siddiqui, ${ }^{1} \mathrm{~J}-\mathrm{K}$ Burkhardt*. ${ }^{1}$ Penn Medicine, Hospital of the University of Pennsylvania, Philadelphia; ${ }^{2}$ Geisinger Medical Center, Danville, PA; ${ }^{3}$ University at Buffalo, State University of New York, Buffalo, NY; ${ }^{4}$ Baptist Stroke and Cerebrovascular Center, Jacksonville, $F L$; ${ }^{5}$ UMASS, 55 Lake Avenue North, Worcester, MA; ${ }^{6}$ Jefferson Health, Philadelphia, PA; 7 University of Miami, Miami, FL, United States

\subsection{6/neurintsurg-2021-ESMINT.61}

Introduction The Walrus Balloon Guide Catheter (BGC) is a new generation of BGC, designed to bypass limitations of conventional BGCs in mechanical thrombectomy.

Objectives To analyze the Walrus BGC for cervical carotid disease (CCD) using the endovascular transcarotid artery revascularization (eTCAR) technique.

Aims Safety and feasibility from a multicenter experience.

Methods Retrospective analysis of prospectively-maintained multicenter datasets

Results 105 patients with high-grade CCD (median carotid stenosis of $83 \%$ ) were included. Navigating the Walrus BGC in the common carotid artery (CCA) was successful in all cases despite type 3/bovine arch anatomy in $26.7 \%$. Emergent treatment for cervical tandem occlusion along with mechanical thrombectomy for acute ischemic stroke was performed in $35.2 \%$ of the cases, with successful recanalization rate (TICI $2 \mathrm{~b} / 3$ ) of $81.1 \%$. Utilizing femoral access in $81.9 \%$ of the patients, carotid stenting was performed in all cases except 4 (angioplasty only); adjunct angioplasty and distal protection devices were used in $86.7 \%$ and in $53.4 \%$ of the cases, respectively. Flow arrest was utilized in the majority of the procedures $(87.6 \%)$, with successful stent deployment achieved in all cases. No major ischemic or Walrus BGC related complications, myocardial infarction or mortality was encountered. Last follow-up mRS of $0-2$ was $70.5 \%$ overall and $98.2 \%$ in elective eTCAR.

Conclusion We present a large multicenter experience of eTCAR technique utilizing the Walrus BGC. In all cases elective or emergent carotid stenting was successful with proximal flow arrest or flow reversal with or without distal protection device with favorable safety profile on follow-up.

Disclosure Jan-Karl Burkhardt, consultant for Q`Apel Medical, Longeviti Neuro Solutions 